\title{
THE DISTINCTION BETWEEN INTRINSIC AND SPURIOUS CONTACT E.M.F.S AND THE QUESTION OF THE ABSORPTION OF RADIATION BY METALS IN QUANTA.
}

\author{
By R. A. Millikan.
}

\author{
SYNOPSIS.
}

\begin{abstract}
Stopping potentials for photo-electrons from $N a, K$ and $L i$ surfaces, as determined with a Faraday cylinder in vacuo for monochromatic ultraviolet light, were found to be accurately the same for all. This result makes general the conclusion that the stopping potential for a given frequency is the same for all clean metals. It is not the same for an oxidized copper surface, however, or for any surface showing a spurious contact e.m.f.

Contact e.m.f.'s between $N a, K$ and $L i$ and the oxidized $C u$ surface of a Faraday cylinder were not found to be constant, but their changes were found to keep pace exactly with changes in the common stopping potential. These changes are attributed to the entanglement of electrons in the copper oxithe surface, thus giving rise to a spurious e.m.f.
\end{abstract}

Intrinsic contact e.m.f. between any two metals is shown to be a definite quantity equal to $\left(h \nu_{0}-h \nu_{0}^{\prime}\right) / e$, when $\nu_{0}$ and $\nu_{0}^{\prime}$ are the threshold frequencies for photoelectric emission from the two metals. This equation enables intrinsic to be distinguished from spurious contact e.m.f. For if the observed contact e.m.f. differs from this value, (which it does not do for clean metals), there is a spurious contact e.m.f. equal to this difference and also equal to the difference in stopping potentials.

The Photo-electric quantity $h \nu_{0}$ is shown to be equal to the thermionic work function pe measured at the same temperature.

Theory of photo-electric emission.-Proof is adduced for the conclusion that either the free, conduction electrons of a metal have the power of taking up the energy $h \nu$ from the incident light, or that, as Barkla has claimed, absorption does not take place in whole quanta. The difficulties associated with each conclusion are pointed out.

\section{INTRODUCTION.}

THE present paper is a continuation of, and a supplement to, one published in 1916, under the title "Einstein's Photoelectric Equation and Contact Electromotive-Force," 1 in which there were pointed out certain necessary conclusions of great importance for the theory of quanta which might be drawn from a given type of photoelectric observations. At that time, however, it had not been possible to make these observations in full and report upon them was therefore deferred, the immediate object there being to obtain evidence for the then unsettled question as to the validity or invalidity of an equation of the type first suggested by Einstein.

1 Phys. Rev., VII., I8, I916. 
In view of the extraordinary sequence of exact determinations of $h$ made with the aid of the Einstein relation, or its inverse suggested earlier by Bragg, a sequence which began with the author's studies in the region of ordinary wave-lengths, ${ }^{1}$ and was continued by those of Duane, Hunt, Hull, and others, in the field of general $x$-radiation, then by those of Webster and others in the field of characteristic x-rays, and finally by those of McLennan, Foote, and a large group of observers in the field of ionizing potentials, we may now set down without fear of error the following equation as representing accurately the facts of observation, whatever may have been, or may now be, its theoretical basis:

$$
\frac{1}{2} M v^{2}=(P D+K) e=h \nu-w=h \nu-h \nu_{0}
$$

in which $v$ is the velocity with which a negative electron escapes from a metallic surface under the influence of a given frequency $\nu$; w the work which it does in getting out of the metal; $\nu_{0}$ the threshold value of the frequency, or the smallest value of $\nu$ which is capable, even with a strong accelerating field (say 20 volts), of detaching a negative electron from the metal at all; $P D$ the stopping potential, or the positive potential which must be applied to the metal to just prevent the escape from it of a photo current, under the influence of the impressed frequency $\nu$, and $K$ the contact e.m.f. between the illuminated metal and the Faraday cylinder into which the liberated electron must escape if it gets away at all.

The introduction of the term $K$ represents an improvement upon Einstein's equation, the necessity for which was first seen, clearly stated, and in large part experimentally justified by Richardson and Compton, ${ }^{2}$ and the correctness of which was very accurately demonstrated by the author, by simultaneous observations in vacuo under identical conditions of contact e.m.f.s, stopping potentials and threshold frequencies (l.c.).

Now if we assume as was done in the I916 paper, and as has in general been done by all workers in the field of the emission of electrons under the influence of ether waves, that the photo-electrons come from the atoms of the metal, an assumption which is apparently justified by the independence of both photo-currents and stopping potentials upon temperature, as well as by the fact that insulators have been shown by the oildrop methods to exhibit a photo-electric behavior precisely like that of conductors, ${ }^{3}$ then the $w$ term in (I) must be made up of two parts $w_{1}$ and $w_{2}$, the former $w_{1}$ representing the work necessary to detach the negative electron from its parent atom, and the latter $w_{2}$ representing

1 Phys. Rev., VII., 355, 1916.

2 Phil. Mag., 24, 592, I9I 2.

${ }^{3}$ Cf. M. I. Kelly, Phys. Rev., I6, 260. 
the work necessary to push the free electron through the surface of the metal. This last term must be precisely the same as that which Richardson has denoted by the product $\varphi e$ in his development of the thermionic equations.

If, now, we push two metals $A$ and $B$ in succession before the Faraday cylinder $F$ (see Fig. I), set up equation (I) with respect to each of them, and subtract, as below, we may make ourselves independent of a measurement of a contact e.m.f. between either metal and the Faraday cylinder. Thus we have

$$
\begin{aligned}
& \left(P D_{A}+K_{A}\right) e=h \nu-h \nu_{0_{A}}=h \nu-\left(w_{1_{A}}+w_{2_{A}}\right) \\
& \left(P D_{B}+K_{B}\right) e=h \nu-h \nu_{0_{B}}=h \nu-\left(w_{1_{B}}+w_{2_{B}}\right)
\end{aligned}
$$

which give, by subtraction

$$
\begin{aligned}
\left(P D_{A}-P D_{B}\right) e+\left(K_{A}-K_{B}\right) e= & \left(w_{1_{B}}-w_{1_{A}}\right) \\
& +\left(w_{2_{B}}-w_{2_{A}}\right)=h \nu_{0_{B}}-h \nu_{0_{A}} .
\end{aligned}
$$

Now, $K_{A}-K_{B}$ is the contact e.m.f. $A_{B}$ (i.e., the contact e.m.f. between the metals $A$ and $B$ ), and by definition $\left(w_{2_{B}}-w_{2_{A}}\right) / e$ is also the contact e.m.f. $A B$. It follows, then, at once from (2), as I pointed out in 19I6, that

and also that

$$
\left(P D_{A}-P D_{B}\right) e=w_{1_{B}}-w_{1_{A}}
$$

$$
\text { Contact e.m.f. } A B=\left(\frac{h \nu_{0_{B}}-h \nu_{0_{A}}}{\boldsymbol{e}}\right)-\left(P D_{A}-P D_{B}\right) .
$$

Equation (4) then, each term of which is capable of direct and independent measurement, must be found satisfied in all cases if Einstein's equation, as modified by Richardson, is correct, and it was subjected to searching experimental test in my former work and found to be always in agreement with observation. The term $h \nu_{0_{A}}$ for freshly cut lithium, for example, was measured by applying a potential of about 20 volts between the lithium and the Faraday cylinder (see Fig. I), the inner surface of which was heavily oxidized so as to render it as light-absorbing as possible, and the wave-length of the incident light was then varied until the threshold frequency was found at $\nu_{0}=57.0 \times \operatorname{Io}^{13}(\lambda=5263 \AA)$. In this experiment, the negative terminal of the 20-volt battery was of course connected to the lithium. The $h \nu_{0_{B}}$ for the copper oxide was then found by reversing the terminal of the battery and varying the wave-length of the light incident upon the lithium until this light, strongly reflected from the lithium surface to the inside of the cylinder, began to cause negative electrons to flow from the cylinder back to the lithium. $\nu_{0_{B}}$ was thus located at $118.2 \times 10^{13} . \quad(\lambda=2535 \AA$. $)$ This 
gave $h / e\left(\nu_{0_{B}}-\nu_{0_{A}}\right)=2.53$ volts. A contact e.m.f. measurement was then made in vacuo by the Kelvin method, by turning the lithium surface through $90^{\circ}$ to the position $A^{\prime}$ (Fig. I) in a manner which has been previously described ${ }^{1}$ and finding directly the potential which had to be applied to just neutralize the contact e.m.f. between it and a copper disc $D$, oxidized in every way as had been the copper Faraday cylinder. This measured contact e.m.f. was in the foregoing case $1.5^{2}$ volts. Now, using line $\lambda=$ 2535, the measured stopping potential for the lithium was found to be I.oo volts, while for the $\mathrm{CuO}$, whose threshold frequency lay at line 2535 , it was of course o. Equation (4) then gave, inserting num-

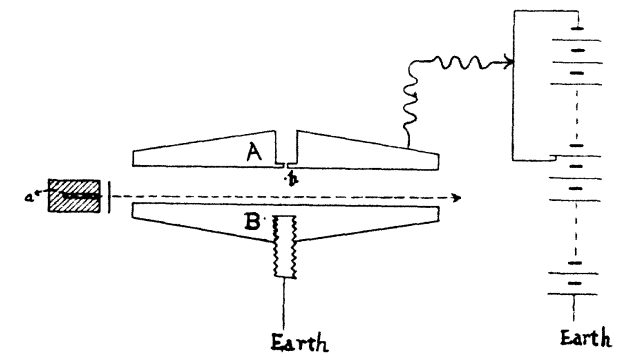

Fig. 1 erical values, $\mathrm{I} .5^{2}=2.53-\mathrm{I} . \mathrm{OO}=\mathrm{I} .53$ which is an identity within the limits of experimental error.

I have thus given again one complete set of observations taken from the preceding work, to show how large the term $\left(P D_{A}-P D_{B}\right)$ often was, in this case a full volt, and to show how impossible it is that in these observations the contact e.m.f. can be given by the simple relation, contact e.m.f. $=h / e\left(\nu_{0_{B}}-\nu_{0_{A}}\right) . \quad I$ have now made many observations by the foregoing method using all three of the metals, sodium, potassium, and lithium, maintained all the time at a vacuum of $10^{-5}$ or lower and although the measured contact e.m.f.s do not in general remain at all constant over long periods of time, creeping in general towards smaller values, and showing variations in a month or two of time amounting to as much as a volt, the changes in the stopping potentials always keep pace with those in contact e.m.f. so that equation (4) has never yet been found to fail. In a word, then, so far as the relations between photoelectric and contact effects are concerned, the demands of Einstein's equation are still found to be fully satisfied.

But, as pointed out in the preceding paper, there have been very strong experimental indications that a contact e.m.f. equation much more limited in scope than (4) actually holds in most cases, namely,

$$
\text { Contact e.m.f. }=\frac{h}{e}\left(\nu_{0_{B}}-\nu_{0_{A}}\right)
$$

and the purpose of the present paper is to present the limits of validity of (5) and the reason for its failure in the above work, and also to discuss

\footnotetext{
${ }^{1}$ Phys. Rev., 7, 362, 19r6.
} 
its significance both with relation to the theory of contact e.m.f.s and the theory of quanta.

\section{Intrinsic and Spurious Contact E.M.F.s.}

Equation (5) was thought by Richardson and Compton ${ }^{1}$ to be consistent with their photoelectric work, but their contact e.m.f.s were either taken from tables or, in certain instances, measured in air, so that the point in question could not be considered as in any way settled by their measurements, since $\left(P D_{A}-P D_{B}\right)$ might well be expected to be small. The most refined and convincing way of testing it should consist in bringing different metals in succession before the same Faraday cylinder, and observing whether, with a given incident frequency, the stopping potential for the different metals is the same; equations (2) and (4) show that if $\left(P D_{A}-P D_{B}\right)=0$ contact e.m.f. must be given by $h / e\left(\nu_{0_{B}}-\nu_{0_{A}}\right)$. This was first done by Millikan and Winchester ${ }^{2}$ in very early photoelectric work, designed primarily to test temperature effects. We did not use monochromatic light, however, and a number of metals were essentially in the same Faraday cylinder at one and the same time, with the consequent possibility of exerting a mutual influence upon one another's fields. The observed differences in stopping potentials, in general small, are not therefore convincing. Page ${ }^{3}$ obtained no differences in stopping potentials when he used freshly scraped surfaces of copper, aluminum and zinc, and Drs. Hennings ${ }^{4}$ and Kadesch in the Ryerson Laboratory, extended Page's conclusion to a considerable number of other metals.

In the I9I6 paper, I left for further study the cause of the unquestionably different result which I had obtained in working with two surfaces one of which was an alkali metal and the other the oxide of copper.

I have now to report a considerable number of further observations, made with the same apparatus, in which all of the alkali metals, $\mathrm{Na}$, $\mathrm{K}$ and $\mathrm{Li}$, were brought in succession before the Faraday cylinder with the result that the stopping potential was always exactly and accurately the same for them all, provided the observations were made at the same time. It made no difference whether one of the metals had recently been shaved in vacuo and the other surface similarly treated weeks earlier, or whether they had all been shaved together. In other words, no surface effects due to residual gases modified the equality of the stopping potentials. The contact e.m.f.s taken at essentially the same time, between each of

${ }^{1}$ Phil. Mag., 24, 592, 1912.

2 Phil. Mag., I4, 201, 1907.

${ }^{3}$ Am. Jr. Sci., 36, p. 501, 1913.

${ }^{4}$ Phys. Rev., 8, 217 , I9I6. 
these three metals and the oxidized copper plate differed among themselves by very appreciable amounts. However, when the apparatus was allowed to stand for some days, or for weeks, the common stopping potential would be found to have changed, in one instance by more than I volt, but it had shifted by the same amount for all the metals, so that the equality between the stopping potentials for the different metals remained exact. Further, the measured contact e.m.f.s were all found to have shifted by precisely the same amount as the stopping potentials. This shows clearly that the change which had taken place was due to the element which was common to all the measurements, namely the heavily oxidized surface of copper. Further, the direction of the change was such as to be consistent with the view that it was due to the charging of the oxide negatively by the simple retention of a swarm of negative electrons by it. The oxide coating acts like a condenser of essentially infinite capacity, but the charge on this condenser varies with the time. This charging up of the copper oxide gives rise to a "spurious contact e.m.f." which was responsible for the finite value of $\left(P D_{A}-P D_{B}\right)$ in the work discussed above. It has no effect upon $\left(\nu_{0_{B}}-\nu_{0_{A}}\right)$ because in the measurement of these threshold frequencies very strong accelerating potentials are externally applied. These completely neutralize any effect of a surface charge. But in the measurement of contact e.m.f. and stopping potential, the surface charge pushes each by the same amount in the direction of negative potentials. This is why the measured contact e.m.f. between sodium and the oxidized plate was at first observed to be about 2.5 volts, but had later fallen to less than .5 volt.

The accompanying experiments, then, taken in connection with those of Page and those of Kadesch and Hennings, show in the most beautiful, quantitative way that all clean metals possess intrinsic contact e.m.f.s and they also establish a criterion by which intrinsic and spurious contact e.m.f.s may be distinguished, namely this:

If equation (5) is found by experiment to be satisfied, only intrinsic contact e.m.f.s are present. If equation (5) is not found to be satisfied, then the difference between the observed contact e.m.f. and $h / e \cdot\left(\nu_{0_{B}}-\nu_{0_{A}}\right)$ is a measure of the presence and amount of a spurious contact e.m.f.

Thus the century-old dispute as to the reality of intrinsic contact e.m.f.s appears to be quite definitely settled by experiments of this type. The experiment of bringing sodium and platinum, for example, in succession before the same Faraday cylinder and finding the stopping photo-potentials exactly the same for the two, despite the fact that the electron leaves the sodium under the influence of a given frequency, (e.g., that corresponding to $\lambda=2535 \AA$. ), with a much higher velocity 
than that with which it leaves the platinum, the threshold frequency for sodium being less than one half its value for platinum, is the most striking proof that each metal creates about itself a contact field which is inversely proportional to the work required to detach the electron from the metal. It is to be emphasized, too, that the result is a purely experimental one which is quite independent of any theory, although it is beautifully in accord with the theory of electron atmospheres.

\section{Significance of the Foregoing Results for the TheOrY of Quanta.}

A finite value, then, of $\left(P D_{A}-P D_{B}\right)$ is merely a measure of the surface charge on the oxide and in no case a measure of the difference in the amounts of work necessary to detach an electron from two different metallic atoms, as is implied in equation (3), since in the case of all clean metals or indeed in the case of metals contaminated with invisible surface films this difference is zero; i.e., $\left(P D_{A}-P D_{B}\right) e=0$ and therefore (cf. (3)) $w_{1_{B}}-w_{1_{A}}=0$. Now, the fact that $w_{1_{B}}-w_{1_{A}}=0$ necessitates one or the other of two alternatives:

I. Either the works necessary to detach an electron from the atom of platinum and the atom of sodium, for example, are the same, or else,

2. The photo-electron which escapes with the highest velocity from a metal under the influence of the incident frequency $\nu$ has exactly the energy $h \nu$ when it arrives at the inner surface of the metal on its way out, no loss of energy $w_{1}$ in getting out of the atom having to be taken into account.

The first of these alternatives is so violently at variance with our definite knowledge of the relative strengths of the bonds which hold the outer electrons to different atoms (cf. the values of ionizing potentials, etc.) that it may at once be dismissed as impossible. If this be admitted we have, then, no alternative but to adopt (2) as the inevitable consequence of the experiments discussed in this report.

These experiments then prove conclusively in any case that at the same temperature there is complete identity between the photoelectric work function $h \nu_{0}$ and the thermionic work function $\varphi$. This identity has always been assumed by Richardson, but the experimental evidence has not heretofore been exact enough to demonstrate the correctness of the assumption. No comparison of experimental values of $h \nu_{0}$ and $\varphi e$ is likely to be as trustworthy as the foregoing evidence.

The significance of (2) for the quantum theory is found in the fact that it, in turn, requires one or the other of the two following alternatives:

(a) Either the incident light is able to take hold of a free electron of the metal and impart to it an energy $h \nu$, or 
(b) The incident light energy is not absorbed in whole quanta at all, but rather absorption continues within the atom until the electron is able to escape from the atom with the energy $h \nu$, the absorption being then $h \nu$ plus $w_{1}, w_{1}$ being the amount required to pull the electron out of the atom.

This last is precisely the conclusion which Barkla has drawn from his measurements upon the ionizing energy of the corpuscular radiations stimulated by characteristic x-rays. ${ }^{1}$ I am not disposed to insist here upon the inevitableness of Barkla's conclusion, but I do wish to insist that the foregoing considerations demand either Barkla's conclusion or else the conclusion that a free electron of a metal is able to absorb energy from an incident light wave and to be endowed by that wave with the energy $h \nu$; for they demand that within the metal there be free electrons which possess the full value of the energy $h \nu$.

Either of the conclusions $(a)$ or $(b)$ required by these experiments is confronted by difficulties. It is very difficult to accept Barkla's conclusion $(b)$ because it means either that there are an infinite number of natural frequencies within a metal, or else that an electron within an atom has the power of absorbing a quantity of energy which is characteristic of itself plus a quantity $h \nu$ which is characteristic only of the incident light. Neither of these assumptions is rationally satisfactory.

On the other hand, the conclusion that the free electrons of the metal take part in the photo-effect would seem to require that stopping potentials diminish with increasing temperature, and also that there be discontinuities in the photo-current potential curve corresponding to the potentials at which the photo-currents due to the bound electrons begin to add their effect to that of the free electrons. Neither of these predictions is as yet in harmony with the facts of observation. It is true that in the experiments of Winchester and the author ${ }^{2}$ upon the effect of temperature upon photo-effects, the temperature was carried through but about $300^{\circ} \mathrm{C}$. in the case of aluminum and about $100^{\circ} \mathrm{C}$. in the case of other metals, and it is possible that a small temperature effect escaped detection. It is also conceivable that a further search for discontinuities in the photo-current potential curve might reveal them. Such a search for discontinuities and for a temperature variation in stopping potentials is now being made.

At any rate, I am at present disposed to adopt alternative $(a)$ and to regard the present experimental work as justifying the conclusion that a light wave has the property of being absorbed by a free electron of a

${ }^{1}$ Phil. Trans., A, Vol. 27, 315-360, 1917.

2 Phil. Mag., 14, 201, 1907. 
metal and of imparting to that electron the energy $h \nu$. If this conclusion is correct it is of much importance for the theory of radiation, for it means that the burden of accounting for the emission of electrons with the energy $h \nu$ can no longer be thrown back upon some unknown mechanism in the structure of the atom where many physicists have in the past sought to place it.

Nor, on the other hand, can this burden be placed upon the elasticsolid properties of the complex of atoms and free (i.e. conduction) electrons constituting a metal. For, although, in view of the Debye and Born types of analysis of the phenomena of specific heats, etc., this complex should possess a practically infinite number of natural frequencies, each of which might get into resonance with the impressed wave of frequency $\nu$, yet the phenomenon of discharging electrons with this energy would then be limited to systems which possess these elastic solid properties, i.e. to solids or at most to liquids. Gases, on the other hand, would be excluded from the possibility of exhibiting it. Yet, though exact quantitative proofs for the case of gases are perhaps not yet available, the evidence yielded by many experiments, including those of C. T. R. Wilson, upon the emission of secondary electrons from the atoms of gases by gamma and $x$ rays is so convincing that it is at present extremely unlikely that gases do not emit electrons with energies which are at least approximately equal to $h \nu$.

If then conclusion " a" above is valid the only remaining alternative is to consider the property of taking hold of an electron, whether free or bound and imparting to it an energy $h \nu$, as an intrinsic property of light itself.

RYERSON PHYSICAL LABORATORY,

University of Chicago. 